\title{
Effects of context on a visual 3-D pointing task
}

\author{
Michelle J A Doumen, Astrid M L Kappers, Jan J Koenderink
}

Helmholtz Institute, Physics of Man, Utrecht University, Princetonplein 5, NL 3584 CC Utrecht,

The Netherlands; e-mail: a.m.I.kappers@phys.uu.nl

Received 22 November 2005, in revised form 22 February 2006; published online 5 January 2007

\begin{abstract}
We examined the effects of egocentric and contextual references on a 3-D exocentric pointing task. Large systematic deviations were found for the slant (angle in the horizontal plane). For most observers, the deviations were smaller when the veridical pointing direction was parallel to a wall. For some observers the size of the deviations was also dependent on whether the veridical pointing direction was frontoparallel or not. For the tilt (angle in the vertical plane), the deviations were smaller and less systematic. Hence, although observers show comparable systematic deviations, the way in which the presence of structure in an environment is used for judging positions of objects is observer-dependent.
\end{abstract}

\section{Introduction}

We know that there are multiple possible sources of information that we can use to see depth (Berkeley 1732; Gibson 1950). However, the fact that these cues are available to observers does not necessarily have to mean that the observers are actually using them (Cutting and Vishton 1995). Hence we need to discriminate between the structure that is available and the information that observers extract from this structure, namely the actual cues for seeing depth. In the research described below, we examined the influence of prominent structure surrounding the setup (eg walls of a room) and the information that observers can extract from internal references.

The term visual space is used in this paper as an operational entity that represents how we visually perceive locations of objects in the world around us. In the early research on visual space, researchers largely ignored the fact that external cues are important. Instead, they focused on egocentric references by using luminous stimuli in a completely dark context (Zajączkowska 1956; Blank 1961). By this method, these researchers found large deviations from veridical settings. This is probably not surprising, since the use of such a paradigm removes a considerable amount of informative structure that is normally present in the visual field.

Other researchers concentrated on contextual information. Ames pointed out that perceptual awareness is dependent on the weighting of the reliability of indications about depth (Ames 1953). These reliabilities are learned through our past experience (Berkeley 1732). With his distorted-room demonstrations (Ittelson 1952), Ames showed that prior knowledge and assumptions that, for instance, walls, windows, and paintings are usually rectangular, can overrule physiological depth cues. An irregularly shaped room could look like a regularly shaped one. If more information is present, ie under free-viewing conditions, this illusion is still present, but is somewhat weaker (Gehringen and Engel 1986).

Yang and Purves (2003) introduced a probabilistic approach that resembles the ideas put forward by Ames. The basis of this approach is that people are experienced observers and make use of prior knowledge (Gibson 1966). Phenomena like the 'specific distance tendency' (Owens and Leibowitz 1976), and the 'equidistance tendency' (Gogel 1965) can be explained by the fact that we are more likely to be surrounded by objects at a certain distance or combination of distances. According to Yang and Purves (2003) 
our brains use Bayesian calculations to let us perceive what is the most probable situation that is possible for a given visual input. Comparing this approach to Ames's theory, we see that both views incorporate prior knowledge. Ames talks about reliabilities of cues, Yang, on the other hand, does not talk about depth cues, but about the likelihood of certain situations. However, both theories incorporate the notion that people can make use of different sources of information, and that the choice of these sources is observer-dependent.

The 'oblique effect' is an effect that is often found in research on visual space (Appelle 1972). It refers to superior performance in tasks involving horizontally or vertically oriented stimuli than in tasks involving obliquely oriented stimuli. Chen and Levi (1996) found oblique effects for retinotopic coordinates with a parallelism discrimination task. Hence, perceptual phenomena like the 'oblique effect' are influenced not only by allocentric references, but also by egocentric ones. For example, Darling and Bartelt (2005) found that an internally specified gravity reference is important for orienting objects visually. Cuijpers et al (2000b) also found an oblique effect in research with a parallelity task in which the observers had to put a bar parallel to a reference bar. The oblique effect that Cuijpers et al found is different from the former oblique effects. First, their research was done in a horizontal plane instead of a frontoparallel plane. Second, Cuijpers et al found larger deviations for oblique orientations than for non-oblique orientations in a parallelity task. However, the conventional literature on the oblique effects describes an increase in the variance of the data instead of a signed deviation. When we refer to the 'oblique effect' in this paper, we mean the effect described by Cuijpers et al, ie an increase in the deviation from veridical settings.

In a second experiment, Cuijpers et al (2001) varied the orientation of the setup with respect to the room, the cabin the observer was seated in, and the orientation of the observer himself. The goal was to measure which references in the environment were causing the oblique effect and thereby the veridicality of the settings of the observers. They concluded that people tend to make use of external references, although they are not consciously aware that they are doing so. The structure that people use in their environment is observer-dependent (Cuijpers et al 2001). Since we found in a pilot experiment that observers use both egocentric and allocentric references for the exocentric pointing task, we examined this idea further.

The task we used in the present experiments was an exocentric pointing task. This task has been used in the horizontal plane at eye-height by several experimenters including Cuijpers et al (2000a), Koenderink et al (2000), and Schoumans et al (2002). During a 2-D exocentric pointing task, an observer has to rotate a pointer with a remote control in the horizontal plane at eye-height. The task is to let it point towards a target. Cuijpers et al (2000a) tried to conceal the information normally provided by walls, floor, and ceiling of the experimental room by covering the walls with wrinkled plastic. In addition, they had the observers seated in a cabin with a chin-rest to prevent them from deriving information from head movements. They found effects of the relative distance between the pointer and the ball and the visual angle. In our earlier experiments the same task and experimental room were used (Doumen et al 2005). The difference with the research of Cuijpers et al was that the observers had an unobstructed view of the walls, ceiling, and floor of the experimental room. Apart from this, the observers could move their heads freely. Our results were remarkably similar to the data of Cuijpers et al (2000a) with regard to the size of the deviations and the structure of the deviations. An interesting aspect of the results of both experiments was that the deviations approached zero when pointer and ball were at the same distance from the observer. We wondered whether this decrement in deviations found for most observers was based solely on the fact that a wall was used as a reference, or whether it could be explained by the fact that these settings were also in the frontoparallel plane. 
Schoumans and colleagues (2002) showed that people use contextual information as a reference for doing an exocentric pointing task within grasping distance. They concluded that observers used both egocentric and allocentric references to judge positions of objects at these distances.

Most research in visual space, including our own, has been concerned with horizontal planes. Therefore, it was a natural step to extend our knowledge to 3-D scenes. To do this, we used a pointer that could rotate both in the horizontal plane and the vertical plane, and could be positioned at different heights. We found a comparable pattern for our 3-D experiments for the settings in the horizontal plane as we found in the previously described 2-D experiments (Doumen et al 2006). The deviations in the vertical plane were smaller than in the horizontal plane and were differently dependent on spatial parameters. In the following experiment we were interested in whether egocentric references and contextual references could contribute to the accuracy and veridicality of the settings of the observers in a 3-D task. Since the use of the structure in the environment in visual tasks is observer-dependent (Cuijpers et al 2001), we expected that the observers would show differences in contextual influences. Furthermore, since oblique effects are common in visual perception, we hypothesised that, in trials where the pointer and the ball were at different heights, the size of the deviations would be larger than in trials where the ball and pointer were at the same height.

In the first experiment we describe below we compared two conditions: one in which the objects were in a plane that was both frontoparallel and parallel to the wall, and the other in which the objects were frontoparallel but not parallel to the wall. In the second experiment, the settings could be either frontoparallel or not and parallel to a wall or not. These two parameters were varied independently of each other.

\section{General methods}

\subsection{Observers}

Fourteen observers participated in our experiments, seven in each of the two experiments. They were paid for their efforts. They all had normal or corrected-to-normal sight and were tested for stereoacuity (all observers had an acuity of more than $60 \mathrm{~s}$ of arc). The observers were tested individually, were naive to the purpose of the experiments, and had little or no experience of participating in psychophysical experiments.

\subsection{Experimental setup}

The experimental room measured $6 \mathrm{~m}$ by $6 \mathrm{~m}$ by $3.5 \mathrm{~m}$ and its top view is shown in figure 1 . The walls of the room were plain white with visible texture. From the ceiling,

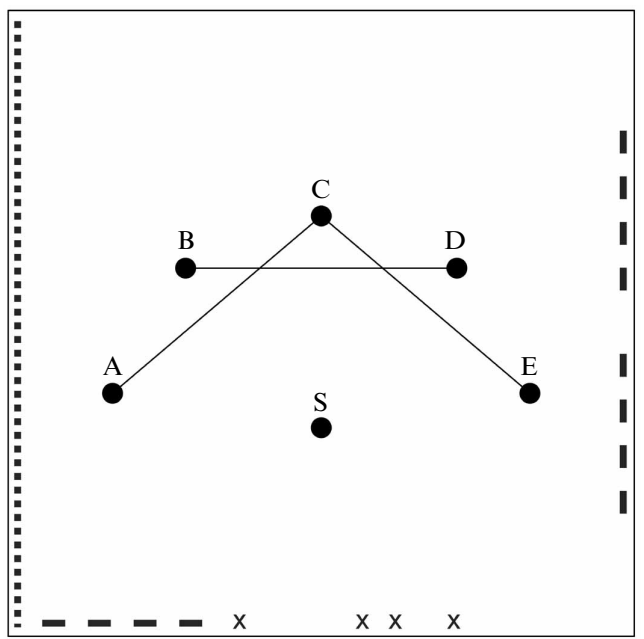

Figure 1. Experiment 1. Top view. The square represents the walls of the room. The dotted line represents a wall with four blinded windows and radiators along the base. The short dashed lines give the positions of the light-gray doors, and the crosses give the positions of four $3 \mathrm{~m}$ high wooden strips that were attached to the wall. The observer was positioned at point $\mathrm{S}$; the objects (pointer and ball) were at positions A through E. The lines between the points represent the pointing directions that were used. 
a horizontal iron grid was suspended below oblong fluorescent lights. The grid was $3 \mathrm{~m}$ above the ground. Green balls that were used as targets were hung from the grid. These balls had a diameter of $6 \mathrm{~cm}$ and could be hung at two heights: $60 \mathrm{~cm}$ and $240 \mathrm{~cm}$ above the ground.

Metal strips were taped on the floor to position the pointer. The strips were visible to the observer. The pointer consisted of a $45 \mathrm{~cm}$ long orange rod connected to a device that contained the motor for rotating it in the vertical plane. The device was covered by a white sphere with a diameter of $14 \mathrm{~cm}$. The pointer stood on a vertical iron rod that was attached to a circularly shaped foot with a motor to generate rotations in the horizontal plane. The motor was concealed by a cylindrical screen. The height of the centre of the pointer was $60 \mathrm{~cm}$. The pointer could be positioned on a pillar so that the total height was $240 \mathrm{~cm}$. The pointer could therefore be positioned at two different heights: 60 and $240 \mathrm{~cm}$ above the ground. The observer could rotate the pointer using two small remote controls: one was for rotating in the horizontal plane, and the other was for rotating in the vertical plane. These remote controls consisted of small joysticks that by themselves gave no information about the orientation of the pointer.

The observer was seated on a revolving chair that could be adjusted in height so that every observer could have an eye-height of $150 \mathrm{~cm}$. This was exactly halfway between the floor and the grid. The two objects - the ball and the pointer-could both be positioned either $90 \mathrm{~cm}$ above or below eye-height, independently of each other.

\subsection{Procedure}

The measurements were done in sessions of $\sim 1 \mathrm{~h}$ each. Before a session began, the height of the chair was adjusted so that the observer was seated with his/her eye-level at the correct height. The observers were instructed to stay seated during the measurements and not to move their upper bodies. They were allowed to rotate their heads or the chair in which they were seated. No references were made where they were allowed to look during the experiment. Furthermore, no feedback was given before the end of the entire experiment. The observer was asked to close his/her eyes when the exact settings of the pointer were read from the device and when the objects were being rearranged for the next trial. Before the observer was allowed to open his/her eyes again, the observer was asked to rotate the pointer randomly in the two directions. This was done to make sure that the observer could not get feedback from the previous trials. At the end of each session the observer was asked to close his/her eyes and rotate the pointer again and the ball was removed from the scene. This way the observer saw only the pointer and the ball together from the position of the chair at an eye-height of $150 \mathrm{~cm}$ above the ground.

\subsection{Analysis}

Two values were collected for each setting: the slant and the tilt. These terms are used differently in various fields of research. Here slant is used as the angle in the horizontal plane (with a range of $360^{\circ}$ ) and tilt is used as the angle in the vertical plane (with a range of $180^{\circ}$ ). We analysed these two values separately. We had three reasons for analysing the data in this way. The first reason was that this way we can compare the data with our previous work with a 2-D task. Second, some authors assume that gravity (and with gravity a distinction between vertical and horizontal directions) is important for orienting (Paillard 1991; Darling and Bartelt 2005). Third, the settings for the slant and the tilt were measured separately (one remote control for each of these two values).

For the slant, a positive deviation means that the observers pointed further away from themselves than the position of the ball (overshoot), whereas a negative deviation means that the observers pointed more towards themselves (undershoot). For the tilt, 
a positive deviation means that the observers pointed too high, a negative deviation means that the observers pointed too low. We conducted analyses of variance for the slant data for the two experiments.

\section{Experiment 1}

\subsection{Methods}

Seven observers participated in this experiment. Four of them were undergraduate students and three were new colleagues (one post-doc and two graduate students). One of the undergraduates was female, all the other observers were male.

Five points were marked on the floor (and the grid) to position the objects. These points were visible, although the points on the raster did not attract much attention. The letters A through E represent these positions in the azimuthal plane (see figure 1). The distances between A and C, B and D, and C and E were $260 \mathrm{~cm}$. The distance from the position of the cyclopean eye of the observer (point $\mathrm{S}$ in figure 1) and these points in the azimuthal plane was $219 \mathrm{~cm}$. Since the observer sat on a revolving chair and all possible positions of the objects were the same distance away from him/her, pointing from $\mathrm{A}$ to $\mathrm{C}$, from $\mathrm{B}$ to $\mathrm{D}$, or from $\mathrm{C}$ to $\mathrm{E}$ could always be in the observer's frontoparallel plane. The difference between the pointing direction $\mathrm{B}-\mathrm{D}$ and the other two pointing directions $(\mathrm{A}-\mathrm{C}$ and $\mathrm{C}-\mathrm{E}$ ) was the presence of a wall parallel to the pointing direction $\mathrm{B}-\mathrm{D}$. The distances from points $\mathrm{B}$ and $\mathrm{D}$ to the back wall were approximately $2.5 \mathrm{~m}$.

Since the objects were at two different heights, there were trials in which the observer had to point upwards, downwards, or horizontally (low or high) - see figure 2.

For the three combinations of points we used the following parameters: the direction of pointing (pointing from left to right and vice versa), the height of the pointer, and the height of the ball. We repeated every condition three times; therefore for each observer we had $3 \times 2 \times 2 \times 2 \times 3=72$ trials (repetitions $\times$ directions $\times$ pointer heights $\times$ ball heights $\times$ combinations of azimuthal positions of the pointer and the ball). We completed the experiment in three sessions of $1 \mathrm{~h}$ each. In each session, all combinations of pointer and ball positions were measured in a random order. Thus, each subsequent session contained the same trials in a different order.

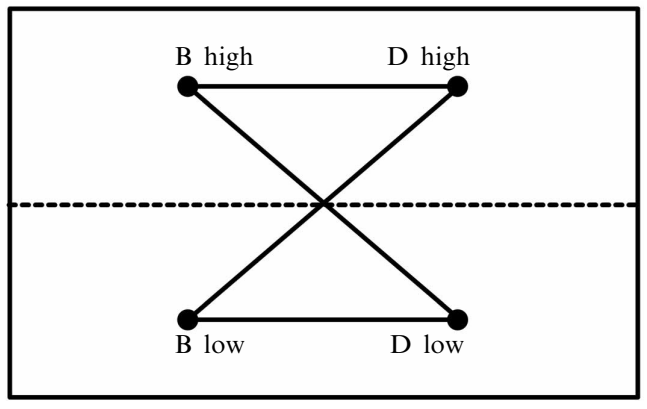

Figure 2. Experiment 1. Frontal view. The dashed line represents the eye-height of the observer ( $1.50 \mathrm{~m}$ above the ground). For each point in the azimuthal plane (in the given example points $\mathrm{A}$ and B) two heights of the objects were used: $60 \mathrm{~cm}$ and $240 \mathrm{~cm}$ above the ground. The lines between the points represent the pointing directions that were used.

\subsection{Results}

3.2.1 Slant. The data for the slant-the deviations in the horizontal plane-are plotted in three graphs in figure 3 . These graphs represent the same data sorted in different ways. The three figures show the deviations from the veridical pointing directions for the seven observers. The standard errors of the mean are also shown. Quite large deviations from veridical settings were found. We found deviations with mean values of up to $17^{\circ}$. The sizes of the deviations were observer-dependent.

A second striking feature of figure 3 is that most values for the slant are positive. This means that the observers almost always overshot the target. More specifically, the 


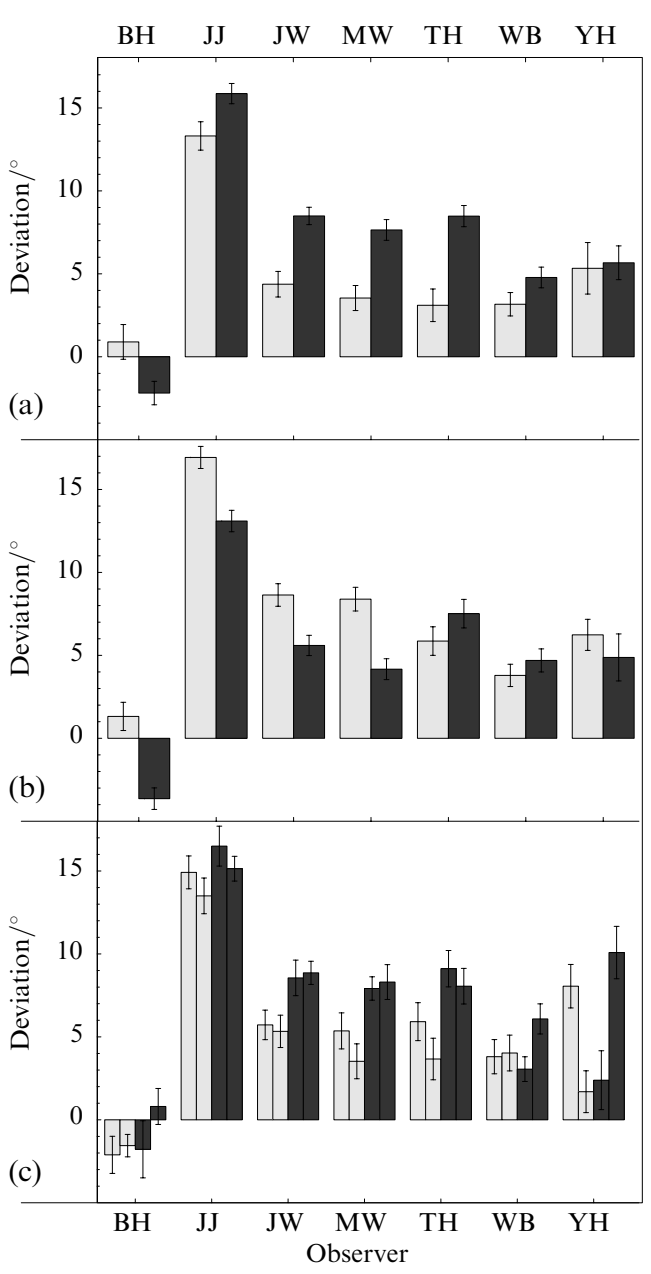

Figure 3. Experiment 1. Slant. Bar charts of the deviations for the slant for each observer in experiment 1 . (a) The grey bars represent the means of the trials that were parallel to the wall, the black bars show the means for the trials not parallel to the wall. (b) The grey bars represent the means of the trials when the observers pointed from left to right, the black bars when they pointed from right to left. (c) The grey bars show the means for pointing horizontally (above eye-height and below eye-height, respectively), the black bars for pointing downwards and upwards.

observers frequently pointed to positions beyond the target. One observer, $\mathrm{BH}$, showed rather small deviations in the slant and these deviations were scattered around zero. He was the only observer who did not show a significant overshooting bias.

Figure 3a shows two bars for each observer. The open bars represent the mean of all trials in which the veridical pointing direction was parallel to the wall. The black bars represent the means of the trials in which the veridical pointing direction was not parallel to the wall. For all observers except $\mathrm{BH}$ the black bars were larger than the open bars, which means that when the wall was parallel to the pointing direction, the deviations of the settings were smaller. In figure $3 \mathrm{~b}$ the open bars represent the trials in which the observer had to point from left to right, the black bars represent the trials in which he/she had to point from right to left. There were differences between the bars for some observers, but these were not all in the same direction. In figure $3 \mathrm{c}$, the open bars represent the trials in which the veridical pointing direction was horizontal (above and below eye-height, respectively). The black bars represent the trials in which the veridical pointing direction was downwards and upwards respectively. For four observers (JJ, JW, MW, and TH) the black bars are larger than the open bars, which means that the deviations from veridical settings were smaller when the observer had to direct the pointer horizontally.

We did an overall analysis of variance with repeated measures. The dependent variable was the deviation of the slant. We used three independent variables: context 
(pointing between $\mathrm{A}$ and $\mathrm{B}, \mathrm{B}$ and $\mathrm{D}$, and $\mathrm{C}$ and $\mathrm{E}$ ), direction (pointing from left to right and vice versa), and height (pointing from a high position to a high position, from high to low, from low to high, and from low to low). We found a trend for both an effect of context and of direction of pointing $(F=3.649, p=0.09$; and $F=4.708$, $p=0.07$, respectively). The deviations were smaller when pointing parallel to the wall and when pointing from right to left. Furthermore, we found an effect of height $(F=5.800, p<0.02)$, with smaller deviations when the pointing direction was horizontal.

Next to the analysis discussed in the previous paragraph, we did an analysis of variance on the slant data for each observer separately. For four observers we found an effect of context, as was expected $(p \mathrm{~s}<0.005)$. A posteriori tests reveal that for these observers pointing between $\mathrm{A}$ and $\mathrm{C}$, and $\mathrm{C}$ and $\mathrm{E}$ (neither parallel to the back wall) did not differ from each other, but there was a difference between these two combinations and B-D (parallel to the wall). These observers-JJ, JW, MW, and TH-thus showed more veridical settings when a wall was parallel to the pointing direction. One observer, $\mathrm{BH}$, showed an effect of context, but it was contrary to the former trend, ie he showed larger deviations when pointing from B to D or vice versa. However, his deviations were rather small and scattered around zero (see figure 3a). Hence, we do not feel justified in concluding that the observer's results were affected by context. The other two observers-WB and $\mathrm{YH}$ - showed no effect of context. See table 1 for $F$ and $p$ values for all observers.

Table 1. The $F$ and $p$ values for the ANOVA of the data of the slant in experiment 1.

\begin{tabular}{|c|c|c|c|c|c|c|c|}
\hline \multirow[t]{2}{*}{ Observer } & \multicolumn{2}{|c|}{ Context } & \multicolumn{2}{|c|}{ Direction } & \multirow{2}{*}{$\begin{array}{l}\text { Height } \\
F_{3,48}\end{array}$} & \multicolumn{2}{|c|}{ Interactions } \\
\hline & $F_{2,48}$ & $p$ & $F_{1,48}$ & $p$ & & $p$ & \\
\hline $\mathrm{BH}$ & 4.795 & $0.013 *$ & 1.974 & 0.130 & 27.444 & $0.000 *$ & \\
\hline $\mathrm{JJ}$ & 4.911 & $0.011 *$ & 2.427 & 0.077 & 23.642 & $0.000 *$ & $\begin{array}{l}\mathrm{H} \times \mathrm{D}, F_{3,48}=4.887, p=0.005 \\
\mathrm{D} \times \mathrm{P}, \quad F_{2,48}=3.716, p=0.032\end{array}$ \\
\hline JW & 16.864 & $0.000 *$ & 7.649 & $0.000 *$ & 20.735 & $0.000 *$ & $\mathrm{D} \times \mathrm{P}, \quad F_{2,48}=5.475, p=0.007$ \\
\hline MW & 15.943 & $0.000 *$ & 10.652 & $0.000 *$ & 37.476 & $0.000 *$ & $\mathrm{D} \times \mathrm{P}, \quad F_{2,48}=4.741, p=0.013$ \\
\hline $\mathrm{TH}$ & 17.808 & $0.000 *$ & 8.065 & $0.000 *$ & 3.784 & 0.058 & $\begin{array}{l}\mathrm{H} \times \mathrm{D}, F_{3,48}=2.949, p=0.042 \\
\mathrm{D} \times \mathrm{P}, F_{2,48}=4.095, p=0.023\end{array}$ \\
\hline WB & 2.280 & 0.113 & 2.775 & 0.051 & 1.348 & 0.251 & $\mathrm{D} \times \mathrm{P}, \quad F_{2,48}=6.044, p=0.005$ \\
\hline YH & 1.051 & 0.357 & 10.812 & $0.000 *$ & 1.163 & 0.286 & $\begin{array}{l}\mathrm{H} \times \mathrm{D}, F_{3,48}=5.947, p=0.002 \\
\mathrm{D} \times \mathrm{P}, F_{2,48}=5.865, p=0.005\end{array}$ \\
\hline
\end{tabular}

Note: For the parameters parallel (P) and height $(\mathrm{H}) \alpha=0.05$ (one-sided). For the parameter direction (D) $\alpha=0.025$ (two-sided); * the slope deviated significantly from 0 .

The direction of pointing did have an effect for four observers- BH, JJ, JW, and MW. All showed the same bias: pointing from left to right resulted in larger deviations of the slant. For $\mathrm{BH}$, however, this was arbitrary because of the positive and negative values of his data.

The height also affected the data for four observers, JW, MW, TH, and YH. A posteriori tests reveal that, when pointing from a high position to a high position and from a low position to a high position, YH had larger deviations than revealed by the data for pointing from high to low and from low to low. The a posteriori tests also showed that there was a difference between the conditions where the veridical pointing direction was horizontal (pointing from high to high and from low to low) and the conditions where it was not horizontal (for JW, MW, and TH). For the other three observers, no effect of height was found. 
3.2.2 Tilt. For the tilt-the deviation from veridical settings in the vertical plane-we found very small deviations (see figure 4), most being less than $5^{\circ}$. No clear pattern emerged from these data. Furthermore, the results did not deviate systematically in one direction as they did for the slant.

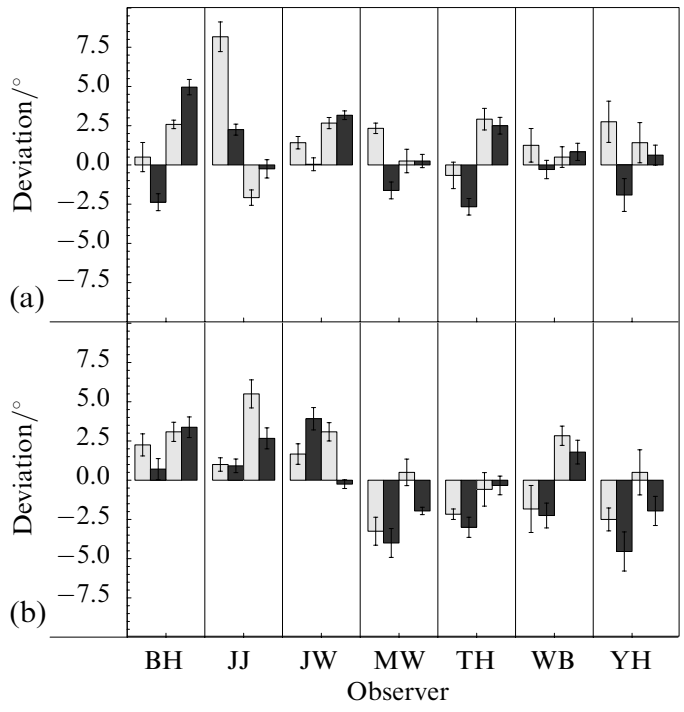

Figure 4. Experiment 1. Tilt. Bar charts of the mean deviations for the tilt for each observer in experiment 1 . The grey bars give the deviations for pointing parallel to the wall, the black bars give the deviations for pointing not parallel to the wall. (a) Data for pointing horizontally: the first two bars for each observer give the data when pointing above eye-height, the second two bars when pointing below eye-height. (b) Data for pointing downwards (the first two bars) and upwards (second two bars).

\subsection{Discussion}

In the horizontal plane, observers mainly overshot when pointing to the ball. This was reported earlier by Cuijpers et al (2000a) and Doumen et al (2005). In those experiments, most deviations for frontoparallel settings were negligible, but the deviations that were found were mostly positive.

We did both an overall analysis and separate analyses for all observers. With the overall analysis we found a trend for an effect of context. This is because some observers show an effect while others do not. Since observer-dependence is quite common with respect to dependence on allocentric references (Cuijpers et al 2001), we think it is more fruitful to look at the individual analyses. In experiment 1 , four out of seven observers showed significantly larger deviations for the slant when pointing parallel to a wall. Three of our observers showed a non-significant difference between the two conditions, while one observer, $\mathrm{BH}$, showed results that differed from those of the other observers (rather small negative deviations). Hence, these results suggest that people differ in the extent to which they use allocentric sources of information to estimate the positions of objects in a scene.

The deviations in the vertical orientation were rather small. This is in line with our previous work (Doumen et al 2006). We found the deviations of the tilt to be smaller than the deviations of the slant, independent of the relative distance. Thus, our first idea that the deviations of the tilt were small because the task could be solved in the visual field, does not hold, since the deviations are also small for settings outside the frontoparallel plane.

For some observers, the deviations in the horizontal orientation are smaller when an external reference is present directly behind the objects. We wanted to know whether these observers also depended on internal references. Therefore we arranged a second experiment in which the setup could be both parallel to a wall or not and frontoparallel or not. This way, we could investigate the possibility of interaction between internal and external references. 


\section{Experiment 2}

\subsection{Methods}

The seven observers who participated in this experiment were all undergraduate students. Four of them were female, the other three male.

Points were marked on the floor and the raster, which lay on a circle with a radius of $130 \mathrm{~cm}$ around the middle of the room (see figure 5). These were the positions of the ball and the pointer in the azimuthal plane. Four positions on the floor were marked for the observer ( $\mathrm{S} 1$ through $\mathrm{S} 4$ ). The azimuthal distance between the observer and the middle of the circle was always $200 \mathrm{~cm}$. For each position of the observer there was always a frontoparallel combination of points on the circle and two different oblique orientations that were at an angle of $45^{\circ}$ to the frontoparallel plane. For each position of the observer, we used two pointing directions in the frontoparallel plane (from left to right and vice versa), and two oblique orientations with the pointer closer to the observer than the ball (from left to right and vice versa). The positions were chosen so that for two positions of the observer (S1 and S3), the frontoparallel combinations were also parallel to one of the walls, and so that for the other two positions of the observer (S2 and S4) the oblique orientations were parallel to one of the walls.

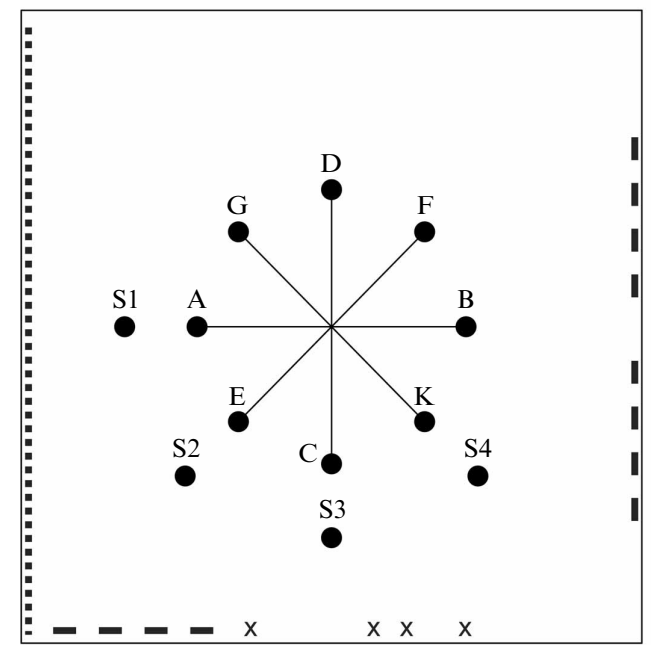

Figure 5. Experiment 2. Top view. The square represents the walls of the room. The dotted line represents a wall with blinded windows and radiators along the base. The short dashed lines give the positions of the doors, and the crosses give the positions of four wooden strips that were attached to the wall. The observer was positioned at points S1 through S4, the objects (pointer and ball) at positions A through $\mathrm{K}$. The lines between the points represent the pointing directions that were used.

The combinations of heights (60 and $240 \mathrm{~cm}$ above the ground) were the same as used in the previous experiment (see figure 2). To do this experiment we therefore needed $4 \times 2 \times 2 \times 4 \times 3=192$ trials (positions of observers $\times$ pointer heights $\times$ ball heights $\times$ combinations of azimuthal positions of pointer and ball $\times$ repetitions). The randomisation of the trials was the same as in experiment 1 . The observer finished all 64 different trials in a random order before starting with the first repetition. After the first repetition, all trials were presented for the third time. Each observer needed approximately $7 \mathrm{~h}$ to complete this experiment, which was done in sessions of $1 \mathrm{~h}$ each.

\subsection{Results}

4.2.1 Slant. As in experiment 1 we found large deviations from veridical settings in the horizontal plane. All deviations (with only a few minor exceptions) were in the same direction: the observer pointed further away than the actual position of the target (overshooting). Figure 6 consists of four bar charts in which the length of the bars represents the size of the deviations in degrees. A positive value means an overshoot, a negative value an undershoot. The standard errors of the mean are also shown.

Figure 6a shows the data for each observer sorted into two groups: parallel to a wall (open bars) and not parallel to a wall (black bars). Most open bars are smaller 


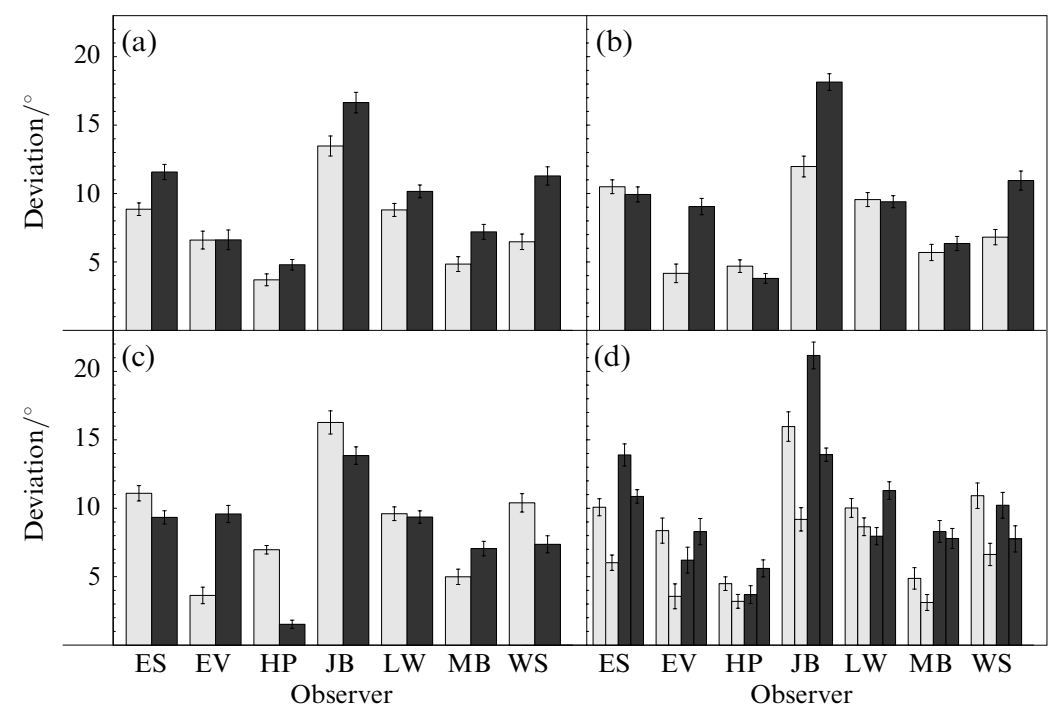

Figure 6. Experiment 2. Slant. Bar charts of the deviations for the slant for each observer in experiment 2. (a) The open bars represent the means of the trials that were parallel to the wall, the black bars show the means for the trials not parallel to the wall. (b) The grey bars show the means of the trials that were frontoparallel, the black bars represent the means for the trials that were not frontoparallel. (c) The grey bars give the means of the trials when the observers pointed from left to right, the black bars when they pointed from right to left. (d) The grey bars represent the means for pointing horizontally (above eye-height and below eye-height, respectively), the black bars for pointing downwards and upwards.

than the black ones, which means that the deviations were smaller when pointing was parallel to a wall. Figure $6 \mathrm{~b}$ shows the same data sorted in a different way, the open bars representing the trials in which the pointing direction was frontoparallel and the black bars representing the trials in which the pointing direction was parallel to a wall. For three observers, EV, JB, and WS, the open bars are smaller than the black ones; thus for these observers pointing in a frontoparallel plane resulted in smaller deviations. Figure 6c gives the data sorted for the pointing direction: pointing from left to right (open bars) and from right to left (black bars). For some observers pointing from left to right yielded more veridical settings, whereas for other observers these settings were achieved by pointing from right to left. Figure $6 \mathrm{~d}$ gives four bars for each observer. The two open bars represent pointing horizontally (above and below eye-height respectively) and the two black bars represent pointing from a high position to a low position and vice versa. The deviations for pointing horizontally are smaller for three observers (ES, JB, and MB).

A repeated-measures analysis over all observers shows an effect of both parallelity to a wall $(F=8.4, p=0.027)$ and frontoparallelity $(F=14.0, p=0.010)$, but not of the direction of pointing (pointing from left to right and vice versa, $F=3.4, p=0.117$ ) and height differences (pointing horizontally or not horizontally, $F=0.3, p=0.615$ ). However, since we can see in figure 6 that there are large differences between observers, we believed it to be more fruitful to analyse the data for each observer separately.

We conducted an analysis of variance on these data for each observer separately. We looked at the effect of parallelity to one of the walls and the effect of frontoparallelity, the effect of pointing direction (from left to right and vice versa) and the difference in height (pointing from a high to a high position, from high to low, from low to high, and from low to low). The combined effects of frontoparallelity and parallelity to a wall suggest four possible results: (i) only frontoparallelity has an influence, 
(ii) only parallelity to a wall has an influence, (iii) frontoparallelity and parallelity to a wall both have an influence, and (iv) neither frontoparallelity nor parallelity to a wall has an influence. Three of these possibilities, but not (iv), applied to our group of seven observers. EV showed only an effect of frontoparallelity. This means that her deviations from veridical settings were smaller when the pointing direction was frontoparallel than when it was not. ES and MB were not affected by this frontoparallelity but showed an effect of the presence of a wall parallel to the pointing direction. For these observers the deviations were smaller when a wall was present parallel to the pointing direction than when there was no wall parallel to this direction. Two observers, JB and WS, showed a statistically significant effect of both parallelity to a wall and frontoparallelity. The final observer, HP, showed an effect of both parallelity to a wall and frontoparallelity, but the weak effect of frontoparallelity was opposite to the trend described above. Hence, we can conclude that for this observer the deviations were smaller when a wall was present parallel to the pointing direction but not when the pointing direction was frontoparallel. See table 2 for the $F$ and $p$ values of all observers. Figures $6 \mathrm{a}$ and $6 \mathrm{~b}$ give a graphical view of these results.

Table 2. The $F$ and $p$ values for the ANOVA of the data of the slant in experiment 2.

\begin{tabular}{|c|c|c|c|c|c|c|c|c|}
\hline \multirow[t]{2}{*}{ Observer } & \multicolumn{2}{|l|}{ Walls } & \multicolumn{2}{|c|}{ Frontoparallel } & \multicolumn{3}{|c|}{ Direction Height } & \multirow[b]{2}{*}{$p$} \\
\hline & $F_{1,160}$ & $p$ & $F_{1,160}$ & $p$ & $F_{1,160}$ & $p$ & $F_{3,160}$ & \\
\hline ES & 23.361 & $0.000 *$ & 1.000 & 0.319 & 7.614 & $0.006 *$ & 33.271 & $0.000 *$ \\
\hline EV & 0.000 & 0.984 & 39.028 & $0.000 *$ & 19.895 & $0.000 *$ & 8.388 & $0.000 *$ \\
\hline HP & 6.300 & $0.013 *$ & 4.147 & $0.043^{*}$ & 78.852 & $0.000 *$ & 5.709 & $0.001 *$ \\
\hline $\mathrm{JB}$ & 23.947 & $0.000 *$ & 90.667 & $0.000 *$ & 1.433 & 0.233 & 58.641 & $0.000 *$ \\
\hline LW & 4.962 & $0.027 *$ & 0.066 & 0.797 & 0.019 & 0.891 & 5.940 & $0.001 *$ \\
\hline $\mathrm{MB}$ & 13.043 & $0.000 *$ & 1.014 & 0.316 & 9.512 & $0.002 *$ & 14.198 & $0.000 *$ \\
\hline WS & 44.943 & $0.000 *$ & 33.186 & $0.000 *$ & 4.801 & 0.030 & 7.942 & $0.000 *$ \\
\hline
\end{tabular}

Note: For the parameters height, walls, and frontoparallel $\alpha=0.05$ (one-sided). For the parameter direction $\alpha=0.025$ (two-sided); * the slope deviated significantly from 0 .

EV, ES, HP, and MB were all affected by the pointing direction. HP and ES had larger deviations from veridical settings when pointing from right to left than vice versa, while EV and $\mathrm{MB}$ had larger deviations when pointing from left to right (see figure 6c). All observers were affected by the height differences. We hypothesised that a difference would be found between trials where the pointer and ball were at different heights and trials where they were at the same height. To test this hypothesis, we added a planned contrast comparison to the analysis. For three observers, ES, JB, and MB, this comparison revealed an overall difference between pointing to a different height and pointing horizontally. A posteriori tests revealed some other differences, but no clear pattern was found among observers (see figure $6 \mathrm{~d}$ for details).

There were many significant interactions. Five of our observers showed an effect of the interaction between height and frontoparallelity; four observers showed an effect of the interaction between parallelity to a wall and frontoparallelity. For three observers we found an interaction effect between direction of pointing and frontoparallelity. See table 3 for the exact values. We will not give further details about these interactions because they do not contribute much to the overall discussion in this paper.

4.2.2 Tilt. With regard to the data of the tilt - the deviation in the vertical direction - the deviations showed positive and negative signs (see figure 7). A positive value means that the observer pointed too high, a negative value means that the setting was below the veridical direction. An analysis of variance is useless for these data because of the signed deviations. Hence, we will look at the data and give an extensive description of our findings. 
Table 3. The $F$ and $p$ values for the interactions found for the ANOVA of experiment 2 .

\begin{tabular}{|c|c|c|c|c|c|c|}
\hline \multirow{2}{*}{$\begin{array}{l}\text { Observer } \\
\mathrm{ES}\end{array}$} & \multicolumn{2}{|c|}{ Effect 1} & \multicolumn{2}{|c|}{ Effect 2} & \multicolumn{2}{|l|}{ Effect 3} \\
\hline & $\mathrm{H} \times \mathrm{A}$ & $\begin{array}{c}F_{3,160}=4.419 \\
\quad p=0.005\end{array}$ & $\mathrm{~A} \times \mathrm{E}$ & $\begin{array}{c}F_{1,160}=6.343 \\
p=0.013\end{array}$ & & \\
\hline EV & $\mathrm{H} \times \mathrm{E}$ & $\begin{array}{c}F_{3,160}=4.469 \\
p=0.005\end{array}$ & $\mathrm{D} \times \mathrm{E}$ & $\begin{array}{c}F_{1,160}=9.447 \\
p=0.002\end{array}$ & $\mathrm{D} \times \mathrm{A} \times \mathrm{E}$ & $\begin{array}{c}F_{1,160}=7.220 \\
p=0.008\end{array}$ \\
\hline HP & $\mathrm{H} \times \mathrm{E}$ & $\begin{array}{c}F_{3,160}=2.913 \\
p=0.036\end{array}$ & $\mathrm{D} \times \mathrm{A}$ & $\begin{array}{c}F_{1,160}=21.541, \\
p=0.000\end{array}$ & $\mathrm{D} \times \mathrm{A} \times \mathrm{E}$ & $\begin{array}{c}F_{1,160}=20.887 \\
\quad p=0.000\end{array}$ \\
\hline$J B$ & $\mathrm{H} \times \mathrm{E}$ & $\begin{array}{l}F_{3,160}=11.950 \\
\quad p=0.000\end{array}$ & $\mathrm{~A} \times \mathrm{E}$ & $\begin{array}{c}F_{1,160}=4.265 \\
p=0.041\end{array}$ & $\mathrm{D} \times \mathrm{E}$ & $\begin{array}{c}F_{1,160}=5.772 \\
p=0.017\end{array}$ \\
\hline LW & $\mathrm{H} \times \mathrm{E}$ & $\begin{array}{c}F_{3,160}=6.288 \\
p=0.000\end{array}$ & $\mathrm{~A} \times \mathrm{E}$ & $\begin{array}{c}F_{1,160}=8.189 \\
p=0.005\end{array}$ & & \\
\hline MB & $\mathrm{H} \times \mathrm{E}$ & $\begin{array}{c}F_{3,160}=10.743 \\
p=0.000\end{array}$ & & & & \\
\hline WS & $\mathrm{D} \times \mathrm{E}$ & $\begin{array}{c}F_{1,160}=6.157 \\
\quad p=0.014\end{array}$ & $\mathrm{~A} \times \mathrm{E}$ & $\begin{array}{c}F_{1,160}=27.899 \\
p=0.000\end{array}$ & $\mathrm{H} \times \mathrm{A} \times \mathrm{E}$ & $\begin{array}{c}F_{3,160}=3.307 \\
\quad p=0.022\end{array}$ \\
\hline
\end{tabular}

Note: Only the interactions with an $\alpha<0.05$ are given. A refers to allocentric references, $\mathrm{E}$ refers to egocentric references, $\mathrm{H}$ refers to height, and $\mathrm{D}$ refers to direction.

Figure 7 gives bar charts for each observer. In both graphs each group of four bars represents the data for all observers. The most striking feature of this pattern is the change in sign that is evident in the first and second black bars of the observers EV, HP, JB, and LW. This means that the deviations were large and opposite in sign when pointing was neither horizontal nor frontoparallel to the observer. When the observers had to point upwards, the deviations were positive, meaning they pointed too high, whereas when the observers had to point downwards, they pointed too low. The other observers do not show such a clear pattern.

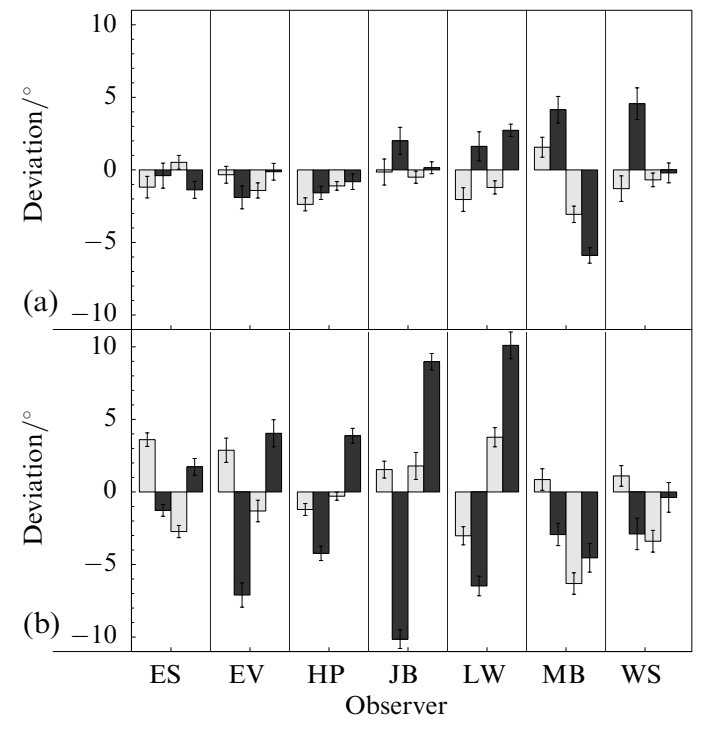

Figure 7. Experiment 2. Tilt. Bar charts of the deviations of the tilt for each observer in experiment 2 . The grey bars show the deviations for pointing frontoparallel, the black bars show the deviations for not pointing frontoparallel. (a) Data for pointing horizontally: the first two bars for each observer represent the data when pointing above eyeheight, the second two bars when pointing below eye-height. (b) Data for pointing downwards (the first two bars) and upwards (second two bars).

\subsection{Discussion}

Regarding the slant, the observers' results revealed large deviations. Positive deviations were found for both pointing in a frontoparallel plane and pointing away from the observer (the pointer was closer to the observer than the ball). The sign of the 
deviations when observers pointed away from themselves corresponded to the results of our 2-D experiments with an exocentric pointing task (Doumen et al 2005). In those experiments we found that the deviations from veridical settings depended on the ratio of the distances of the two objects (ball and pointer) from the observer. When the pointer was further away from the observer than the ball, the deviations reflected an undershot, while when the pointer was closer to the observer, the deviations reflected an overshot. This effect was also found by Cuijpers and colleagues (2000a). However, the positive deviations for the frontoparallel settings, where the ratio between the distances from observer to objects was 1, were negligible in our previous work but in the present experiments we also found large deviations for frontoparallel settings.

Furthermore, we examined whether there was an effect of height difference in the sense that we hypothesised that the deviations from veridical settings would be smaller when the veridical pointing direction was horizontal. For three observers, we found smaller deviations for the slant when the veridical pointing direction was horizontal. For the other observers we found no difference between pointing horizontally and not horizontally for the data of the slant.

For four observers we found that the pointing direction affected the slant data, ie a difference in pointing from left to right and vice versa. This effect was found for both directions (pointing from left to right and from right to left) and could not be explained by the structure provided by the walls. Each condition was measured in two different orientations in the room, thus with two different backgrounds. This way, the structure on the walls surrounding the setup varied between the conditions. For position S1 (see figure 5), the wall directly in front of the observer contained two light-gray doors, whereas for position S3 the wall in front of the observer contained no doors or windows. For the positions S2 and S4 one of the walls that was visible when the observer looked straight ahead was the white wall, and the other was a wall with doors or windows. For position S2 the wall with more structure was on the right, while for position S4 the wall with more structure was on the left. Since the data for the two corresponding positions (S1 and S3, S2 and S4) were combined and the effect of pointing direction is present in both directions, we believe these effects of pointing direction are due to personal biases.

We found some effects of interaction between parameters in the analysis. One of the most salient interaction effects that we found was the interaction between frontoparallelity and parallelity to a wall in the case of observers WS, LW, ES, and JB. We can conclude from the data for these observers that the deviations were smallest when the settings were both frontoparallel and parallel to one of the walls.

We found smaller deviations for the tilt than for the slant. For the frontoparallel settings, the same random pattern was found as in experiment 1 . In the conditions oblique with respect to the observer, we found larger deviations for some observers. For four of our observers the size of these deviations was also dependent on the height differences. Thus, if the settings are frontoparallel and horizontal, the deviations of the tilt are small, but if the settings are oblique with respect to the observer and not horizontal, then the deviations are larger. So in these trials we found an 'oblique effect' with regard to the size of the deviations. This is in contrast with the oblique effect that is most often discussed in the literature (Appelle 1972), concerning the variance of the data. The 'oblique effect' we found was especially prominent with regard to egocentric references, which is in agreement with the results reported in the work of Chen and Levi (1996) and Darling and Bartelt (2005). 


\section{General discussion and conclusions}

The first important point we want to make is that large deviations from veridical settings were found with the 3-D exocentric pointing paradigm. For the slant, deviations of up to $20^{\circ}$ were found. When comparing these results to results obtained with a 2-D exocentric pointing task (Doumen et al 2005), we can conclude that the spread of the data is larger for the 3-D task. This might reflect the increased level of difficulty in this task that involved an extra degree of freedom, namely the orientation in the vertical plane. The sizes of the deviations are in agreement with our previous work with the 3-D exocentric pointing task (Doumen et al 2006).

Looking at the data for the slant, we see that most observers overshoot when the pointing direction is frontoparallel. When we compare this result to the results of research by Koenderink and van Doorn (1998), we find the same pattern even though that research was done outdoors while ours was done indoors. At least for frontoparallels that are closer than $4 \mathrm{~m}$ from the observers, the perceived frontoparallels were all concave towards the observer. For this distance this effect is irrespective of the task that is given to the observer. Koenderink et al (2002), for example, used a line bisection task and found comparable results at these distances.

Overshooting was also found in the conditions where the pointer was closer to the observer than the ball (experiment 2). This replicates the results of our 2-D experiments in which we used an exocentric pointing task, and varied the relative distance of pointer and ball to the observer (Doumen et al 2005).

We found much smaller deviations from veridical settings for the tilt than for the slant. The deviations rarely exceeded $5^{\circ}$, particularly when the veridical settings were in the frontoparallel plane. In the frontoparallel trials, the task can be solved in the visual field, ie the coordinates of the retina correspond to the coordinates in this plane, which makes the task less difficult. When the veridical orientation was oriented obliquely towards the observer (experiment 2) and not horizontally, the deviations were larger for most observers. For four observers we found a pattern that involved pointing too high when pointing downwards and pointing too low when pointing upwards. Hence, for these observers visual space seems to be compressed in the vertical orientation. We could link this to the idea that visual space seems 'stretched' in the horizontal plane (Doumen et al 2005). When visual space is 'stretched' in the horizontal direction, the perceived tilt will be smaller than the physically veridical tilt.

The most salient question posed in this paper concerns the types of information people use when doing an exocentric pointing task. To obtain some insight into this issue, we let observers point parallel to a physical (allocentric) reference, a wall parallel to the pointing direction, and to an egocentric reference, a frontoparallel plane. In the first experiment, all trials were in the frontoparallel plane, but only one condition had pointing orientations parallel to a wall. In this experiment we found that four out of seven observers were influenced by the presence of the wall. This means that the deviations from veridical settings were smaller when the observers pointed parallel to the wall. We could say that these people were able to use the structure provided in order to perform the task more veridically. In the second experiment, where we varied both the allocentric and the egocentric references, six out of seven observers showed smaller deviations when there was a wall parallel to the pointing direction. This experiment gives us information not only about the use of allocentric references but also about the egocentric references we discussed above. The observer who was not influenced by the walls used this egocentric information. Two other observers were also influenced by this egocentric reference, so these observers were influenced by both egocentric and allocentric references provided during the task. This result partly contradicts the results of Cuijpers et al (2001). All their observers were influenced by allocentric references, although which structure of the visual scene was used the cabin 
or the wall of the room) was observer-dependent. However, they found none of their observers to be dependent on egocentric references. This difference could be due to the fact that the observers used by Cuijpers et al were restricted in their head movements by a chin-rest. But it could also be due to the different task used (a parallelity task instead of an exocentric-pointing task). In our earlier work, we also found differences between settings for a parallelity task and an exocentric-pointing task. We believe that this could be because for an exocentric-pointing task an observer needs to make a judgment about the positions of both the pointer and the ball, whereas for a parallelity task the observer does not have to know the actual positions of the two rods in order to do the task. If the positions of the two objects are judged by their relative distances from the observer, it is not surprising to find that observers depended on egocentric references. Furthermore, following this line of reasoning, it is not surprising that no such dependence was found in the experiments of Cuijpers and colleagues, that were done with a parallelity task.

From our experiments we can conclude that people make a personal selection of the sources of information they use for judging depth in a scene. This set of useful sources is probably based on the prior experiences and knowledge of each individual. We cannot yet say whether this involves a weighting up or a selection of these different sources, or whether the knowledge is based on a statistical process or on the weighting up of reliabilities of cues. Of course, we should be careful with making generalisations to other situations and experimental setups. However, in our experiments, we found effects of contextual information, and we believe that our setup is not a special case. Thus we assume that our results can be generalised to other conditions. Therefore, we know that there are differences between observers and that both information from the context (allocentric references) and information from the observer himself/herself (egocentric information) are important for judging the positions of objects.

Acknowledgments. The investigations were supported by the Research Council for Earth and Life Sciences (ALW) with financial aid from the Netherlands Organization for Scientific Research (NWO).

\section{References}

Ames A, 1953 "Reconsideration of the origin and nature of perception in situations involving only inorganic phenomena", in Vision \& Action Ed. S Ratner (Rutgers, NJ: Rutgers University Press) pp $251-274$

Appelle S, 1972 "Perception and discrimination as a function of stimulus orientation: the 'oblique effect' in man and animals" Psychological Bulletin 78 266-278

Berkeley G, 1732 An Essay Towards a New Theory of Vision 4th edition. Retrieved 21 September 2004 from http://psychclassics.yorku.ca

Blank A A, 1961 "Curvature of binocular visual space. An experiment" Journal of the Optical Society of America 51335 - 339

Chen S, Levi D M, 1996 "Meridional anisotropy in the discrimination of parallel and perpendicular lines-effect of body tilt" Perception $25633-649$

Cuijpers R H, Kappers A M L, Koenderink J J, 2000a "Investigation of visual space using an exocentric pointing task" Perception \& Psychophysics 62 1556-1571

Cuijpers R H, Kappers A M L, Koenderink J J, 2000b "Large systematic deviations in visual parallelism" Perception 291467 - 1482

Cuijpers R H, Kappers A M L, Koenderink J J, 2001 "On the role of external reference fames on visual judgements of parallelity" Acta Psychologica 108 283-302

Cutting J E, Vishton P M, 1995 "Perceiving layout and knowing distances: the integration, relative potency, and contextual use of different information about depth", in Perception of Space and Motion Eds W Epstein, S J Rogers (San Diego, CA: Academic Press) pp 69-117

Darling W G, Bartelt R, 2005 "The visual perception coordinate system uses axes defined by the Earth, trunk, and vision" Perception $3417-30$

Doumen M J A, Kappers A M L, Koenderink J J, 2005 "Visual space under free viewing conditions" Perception \& Psychophysics $671177-1189$

Doumen M J A, Kappers A M L, Koenderink J J, 2006 "Horizontal-vertical anisotropy in visual space" Acta Psychologica $123219-239$ 
Gehringen W L, Engel E, 1986 "Effect of ecological viewing conditions on the Ames' distorted room illusion" Journal of Experimental Psychology 12181 - 185

Gibson J J, 1950 The Perception of the Visual World (Cambridge, MA: Riverside Press)

Gibson J J, 1966 The Senses Considered as Perceptual Systems (Boston, MA: Houghton Mifflin)

Gogel W C, 1965 "Equidistance tendency and its consequences" Psychological Bulletin 64 153-163

Ittelson W H, 1952 The Ames Demonstrations in Perception (Princeton, NJ: Princeton University Press)

Koenderink J J, Doorn A J van, 1998 "Exocentric pointing", in Vision and Action Eds L R Harris, M Jenkin (Cambridge: Cambridge University Press) pp 295-313

Koenderink J J, Doorn A J van, Kappers A M L, Lappin J S, 2002 "Large-scale visual frontoparallels under full-cue conditions" Perception 31 1467-1475

Koenderink J J, Doorn A J van, Lappin J S, 2000 "Direct measurement of the curvature of visual space" Perception $2969-79$

Owens D A, Leibowitz H W, 1976 "Oculomotor adjustments in darkness and the specific distance tendency" Perception \& Psychophysics $202-9$

Paillard J, 1991 "Motor representational framing of space", in Brain and Space Ed. J Paillard (Oxford: Oxford University Press) pp $163-182$

Schoumans N, Kappers A M L, Koenderink J J, 2002 "Scale invariance in near space: pointing under influence of context" Acta Psychologica 110 63-81

Yang Z, Purves D, 2003 "A statistical explanation of visual space" Nature Neuroscience $6632-640$

Zajączkowska A, 1956 "Experimental determination of Luneburg's constants $\sigma$ and $K$ " Quarterly Journal of Experimental Psychology 8 66-78 


\section{PERTEPTION}

VOLUME 362007

www.perceptionweb.com

Conditions of use. This article may be downloaded from the Perception website for personal research by members of subscribing organisations. Authors are entitled to distribute their own article (in printed form or by e-mail) to up to 50 people. This PDF may not be placed on any website (or other online distribution system) without permission of the publisher. 